\title{
ANALYTICAL MODELS FOR THE COMPUTATION OF ERROR PROBABILITY OF MULTI-LEVEL PHASE SHIFT KEYING MODULATION SCHEME
}

\section{Isaac A. Ezenugu ${ }^{1}$ and Dike Happiness Ugochi}

Department of Electrical/Electronic Engineering, Imo State University (IMSU), Owerri, Nigeria. E-mail: isaac.ezenugu@yahoo.com

Department of Electrical/Electronic Engineering, Imo State University (IMSU), Owerri, Nigeria

Cite this article:

Isaac A. E., Dike H.U. (2021), Analytical Models for the Computation of Error Probability of Multi-Level Phase Shift Keying Modulation Scheme. British Journal of Computer,

Networking and Information Technology 4(1), 13-20. DOI: 10.52589/BJCNIT-

\section{LSFCMVLI.}

\section{Manuscript History}

Received: 6 March 2021

Accepted: 1 April 2021

Published: 30 June 2021

Copyright $\odot 2020$ The Author(s). This is an Open Access article distributed under the terms of Creative Commons AttributionNonCommercial-NoDerivatives 4.0 International (CC BY-NC-ND 4.0), which permits anyone to share, use, reproduce and redistribute in any medium, provided the original author and source are credited.
ABSTRACT: In this paper, analytical models for the computation of error probability (BER) of the Multi-level Phase Shift Keying (MPSK) modulation scheme is presented. Analytical models for computing MPSK bit error probability based on $Q$ function, error function (erf) and complementary error function (erfc) are presented. Also, an analytical model for computing the symbol error rate for MPSK is presented. Furthermore, a generalized analytical expression for BER as a function of modulation order $(M)$ and energy per bit to noise power density ratio (Eb/No) is presented. The BER was computed for various values of $M(2 \leq M \leq 256)$ and $E b / N o(0 d B \leq E b / N o \leq 14 \mathrm{Db})$. The results showed that at Eb/No $=12 \mathrm{~dB}$, a BER of 9.006E-09 is realized for $M=2$ and $M=4$ whereas BER of 1.056E-01 is realized for $M=256$. Also, for the same $M=2$, the value of $B E R$ decreased from 1.2501E-02 at Eb/No $=4 \mathrm{~dB}$ to 9.0060E-09at $E b / N o=12 \mathrm{~dB}$. Generally, the results showed that for the MPSK modulation scheme, for a given value of Eb/No, the lower modulation order (M) has a lower BER and for a given modulation order, $(M)$ the BER decreases as Eb/No increases.

KEYWORDS: Error Probability, Phase Shift Keying, Q function, Error Function (erf), Complementary Error Function (ERFC), Symbol Error Rate 


\section{INTRODUCTION}

Phase shift keying (PSK) is a form of digital modulation scheme in which data is conveyed by altering the phase of the carrier wave $[1,2,3,4,5]$. In practice, there are so many ways that the carrier signal phase can be altered to represent data and this gives rise to different forms of PSK modulation schemes. The binary phase-shift keying (BPSK) is the simplest form of PSK where there are two possible phases for the carrier signal, where logic 1 data is resented by one phase and logic 0 is represented by the other phase $[6,7,8,9,10,11]$. Consequently, as the incoming digital signal state changes state from 0 to 1 the outgoing carrier signal phase shifts between two angles which are separated by $180^{\circ}$.

On the contrary, in the Multi-level Phase Shift Keying (MPSK ) modulation scheme, instead of two-phase variations, multiple phase variations are used to represent the digital data. Specifically, in BPSK only one bit $(n=1)$ is represented, however in MPSK there is M number of different signal phases that are used to represent $\mathrm{n}$ bits of digital data, where $\mathrm{n}=(M)$ $[12,13,14,15,16,17]$.

Generally, the MPSK modulation scheme affords a higher data rate along with enhanced bandwidth efficiency $[18,19,20]$. Consequently, it is considered one of the most proficient digital data transmission scheme. The BPSK and different forms of QPSK are used in wireless communication, mobile communication, deep space telemetry and satellite communication. In this paper, analytical models for the computation of error probability of Multi-Level Phase Shift Keying (MPSK) modulation scheme is presented. Particularly, the different analytical approaches that can be used to determine the Bit Error Probability (PEB) and Symbol Error Probability (SEB) of the MPSK modulation scheme are presented. Numerical examples are also presented.

\section{ANALYTICAL MODEL FOR MPSK BIT ERROR PROBABILITY AND SYMBOL ERROR PROBABILITY}

\section{MPSK BIT Error Probability}

The Q function-based analytical model for computing MPSK bit error probability, denoted as $P_{b M-P S K}(Q f n)$ is given as;

$$
\begin{array}{r}
P_{b M-P S K}(Q)=\left(\frac{2}{(M)}\right) Q\left(\operatorname{Sin}\left(\frac{\pi}{M}\right) \sqrt{\left(2((M))\left(\frac{\varepsilon_{b}}{N_{0}}\right)\right)}\right) M>4 \\
P_{b M-P S K}(Q)=Q\left(\sqrt{2\left(\frac{\varepsilon_{b}}{N_{0}}\right)}\right) \text { for } M=2 \text { and } M=4
\end{array}
$$

where $\mathrm{M}$ is the modulation order, $N_{0}$ is the noise power density and $\varepsilon_{b}$ is the energy per bit.

The complementary error function (erfc)-based model for computing MPSK bit error probability, denoted as $P_{b M-P S K}(\operatorname{erf} c)$, is given as; 


$$
\begin{gathered}
P_{b M-P S K}(\operatorname{erfc})=\left(\frac{1}{(M)}\right) \operatorname{erfc}\left(\operatorname{Sin}\left(\frac{\pi}{M}\right) \sqrt{\left(((M))\left(\frac{\varepsilon_{b}}{N_{0}}\right)\right)}\right) \text { for } M>4 \\
P_{b M-P S K}(\operatorname{erf} c)=\left(\frac{1}{2}\right) \operatorname{erfc}\left(\sqrt{\frac{\varepsilon_{b}}{N_{0}}}\right) \text { for } M=2 \text { and } M=4
\end{gathered}
$$

The error function (erf)-based MPSK bit error probability, denoted as $P_{b M-P S K}(\operatorname{erf})$, is given as;

$$
\begin{gathered}
P_{b M-P S K}(\operatorname{erf})=\left(\frac{1}{(M)}\right)\left(1-\operatorname{erf}\left(\operatorname{Sin}\left(\frac{\pi}{M}\right) \sqrt{\left(((M))\left(\frac{\varepsilon_{b}}{N_{0}}\right)\right)}\right)\right) \text { for } M>4 \\
P_{b M-P S K}(\operatorname{erf})=\left(\frac{1}{2}\right)\left(1-\operatorname{erf}\left(\sqrt{\frac{\varepsilon_{b}}{N_{0}}}\right)\right) \text { for } M=2 \text { and } M=4
\end{gathered}
$$

\section{MPSK Symbol Error Probability}

The energy per symbol $\left(\varepsilon_{s}\right)$ and the energy per bit $\left(\varepsilon_{b}\right)$ are related as follows;

$$
\varepsilon_{s}=\varepsilon_{b}((M))
$$

Hence, the MPSK modulation probability of symbol error using the Q function is denoted as $P_{S M-P S K}(Q)$, where;

$$
P_{S M-P S K}(Q)=P_{b M-P S K}(Q)((M))=(2) Q\left(\operatorname{Sin}\left(\frac{\pi}{M}\right) \sqrt{\left(2\left(\frac{\varepsilon_{S}}{N_{0}}\right)\right)}\right) \quad M>4
$$

Similarly, using the erfc function, MPSK modulation probability of symbol error, denoted as $P_{S M-P S K}(\operatorname{erfc})$ is given as;

$$
P_{S M-P S K}(\operatorname{erfc})=\operatorname{erfc}\left(\operatorname{Sin}\left(\frac{\pi}{M}\right) \sqrt{\frac{\varepsilon_{S}}{N_{0}}}\right) \text { for } M>4
$$

Again, using the erf function, the MPSK modulation probability of symbol error, denoted as $P_{S M-P S K}(\operatorname{erf})$ becomes;

$$
P_{S M-P S K}(\operatorname{erf})=1-\operatorname{erf}\left(\operatorname{Sin}\left(\frac{\pi}{M}\right) \sqrt{\frac{\varepsilon_{S}}{N_{0}}}\right) \text { for } M>4
$$

\section{Generalized Formula for Computing MPSK Modulation Probability Of BIT Error}

A generalized formula for computing the MPSK modulation probability of bit error using the erfc function is expressed as; 


$$
P_{b M-P S K}(\operatorname{erfc})=\left(A_{\text {erfc }}\right) \operatorname{erfc}\left(\left(C_{\text {erfc }}\right) \sqrt{\left(B_{\text {erf } c}\right)\left(\frac{\varepsilon_{b}}{N_{0}}\right)}\right)
$$

Where

$$
\begin{array}{r}
A_{\text {erfc }}=\frac{1}{(M)} \\
B_{\text {erf } c}=(M) \\
C_{\text {erfc }}=\operatorname{Sin}\left(\frac{\pi}{M}\right) \quad \text { where } \frac{\pi}{M} \text { is in radians }
\end{array}
$$

Notably, the MPSK modulation probability of bit error for $\mathrm{M}=2$ and $\mathrm{M}=4$ are equal and they are denoted as $P_{b 2-P S K}(e r f c)$ and $P_{b 4-P S K}(\operatorname{erfc})$ respectively and are given as;

$$
P_{b 2-P S K}(\operatorname{erfc})=P_{b 4-P S K}(\operatorname{erfc})=\left(\frac{1}{2}\right) \operatorname{erfc}\left(\sqrt{\left(\frac{\varepsilon_{b}}{N_{0}}\right)}\right)
$$

\section{RESULTS AND DISCUSSION}

The generalized formula was used to compute the MPSK modulation probability of bit error (BER) for modulation order ranging from 2 to 256 and for Eb/No values in the range of $0 \mathrm{~dB}$ to $24 \mathrm{~dB}$. The results for the model parameters, Aerfc, Berfc and Cerfc are shown in Table 1 while Table 2 and Figure 1 show the BER for the various Eb/No and modulation order.

Also, the results of the BER versus modulation order for various values of Eb/No are shown in Table 3 and Figure 2.

The results show that at $\mathrm{Eb} / \mathrm{No}=12 \mathrm{~dB}$, a BER of $9.006 \mathrm{E}-09 \quad$ is realized for $\mathrm{M}=2$ and $\mathrm{M}=4$ whereas $\mathrm{BER}$ of $1.056 \mathrm{E}-01$ is realized for $\mathrm{M}=256$. Also, for the same $\mathrm{M}=2$, the value of BER decreased from 1.2501E-02 at $\mathrm{Eb} / \mathrm{No}=4 \mathrm{~dB}$ to $9.0060 \mathrm{E}-09$ at $\mathrm{Eb} / \mathrm{No}=12 \mathrm{~dB}$ (as shown in Table 2 and Table 3). Generally, the results showed that for the MPSK modulation, for a given value of Eb/No, the lower modulation order $(\mathrm{M})$ has lower BER and for a given modulation order, (M) the BER decreases as Eb/No increases (as shown in Figure 1 and Figure 2).

Table 1: Values of Aerfc, Berfc and Cerfc for the MPSK probability of bit error using the generalized formula based on error function ( $e r f c$ )

\begin{tabular}{|c|c|c|c|c|}
\hline \multicolumn{5}{|c|}{$P_{b M-P S K}(e r f c)=\left(A_{\text {erfc }}\right) \operatorname{erfc}\left(\left(C_{\text {erfc }}\right) \sqrt{\left(B_{\text {erfc }}\right)\left(\frac{\varepsilon_{b}}{N_{0}}\right)}\right)$} \\
\hline Modulation Order, M & K (Bits Per Symbol) & Aerfc & Berfc & Cerfc \\
\hline 2 & 1 & $1 / 2$ & 1 & 1.0000 \\
\hline 4 & 2 & $1 / 2$ & 1 & 1.0000 \\
\hline 8 & 3 & $1 / 3$ & 3 & 0.3827 \\
\hline
\end{tabular}


British Journal of Computer, Networking and Information Technology

ISSN: 2689-5315

Volume 4, Issue 1, 2021 (pp. 13-20)

www.abjournals.org

\begin{tabular}{|c|c|c|c|c|}
\hline 16 & 4 & $1 / 4$ & 4 & 0.1951 \\
\hline 32 & 5 & $1 / 5$ & 5 & 0.0980 \\
\hline 64 & 6 & $1 / 6$ & 6 & 0.0491 \\
\hline 128 & 7 & $1 / 7$ & 7 & 0.0245 \\
\hline 256 & 8 & $1 / 8$ & 8 & 0.0123 \\
\hline
\end{tabular}

Table 2 The MPSK BER versus Eb/No (dB)

\begin{tabular}{|c|c|c|c|c|c|c|c|c|}
\hline \begin{tabular}{|c} 
Modula \\
tion \\
Order, \\
M
\end{tabular} & 2 & 4 & 8 & 16 & 32 & 64 & 128 & 256 \\
\hline $\begin{array}{c}\mathrm{K} \\
\text { (bits/sy } \\
\text { mbol) } \\
\end{array}$ & 1 & 2 & 3 & 4 & 5 & 6 & 7 & 8 \\
\hline $\begin{array}{c}\mathrm{Eb} / \mathrm{No} \\
(\mathrm{dB})\end{array}$ & \begin{tabular}{|l|} 
MPSK \\
BER \\
For \\
M=2 \\
\end{tabular} & \begin{tabular}{|l} 
MPSK \\
BER \\
For \\
M=4 \\
\end{tabular} & \begin{tabular}{|l} 
MPSK \\
BER \\
For \\
M=8 \\
\end{tabular} & $\begin{array}{l}\text { MPSK } \\
\text { BER For } \\
\text { M=16 } \\
\end{array}$ & \begin{tabular}{|l} 
MPSK \\
BER For \\
M=32 \\
\end{tabular} & \begin{tabular}{|l} 
MPSK \\
BER For \\
M=64 \\
\end{tabular} & \begin{tabular}{|l} 
MPSK \\
BER For \\
M=128 \\
\end{tabular} & \begin{tabular}{|l} 
MPSK \\
BER For \\
M=256 \\
\end{tabular} \\
\hline 0 & $\begin{array}{r}7.865 \mathrm{E}- \\
02 \\
\end{array}$ & $\begin{array}{r}7.865 \mathrm{E}- \\
02 \\
\end{array}$ & $\begin{array}{r}1.162 \mathrm{E}- \\
01 \\
\end{array}$ & $\begin{array}{r}1.453 \mathrm{E}- \\
01 \\
\end{array}$ & $\begin{array}{r}1.513 \mathrm{E}- \\
01 \\
\end{array}$ & $\begin{array}{r}1.442 \mathrm{E}- \\
01 \\
\end{array}$ & $\begin{array}{r}1.324 \mathrm{E}- \\
01 \\
\end{array}$ & $\begin{array}{r}1.201 \mathrm{E}- \\
01 \\
\end{array}$ \\
\hline 2 & $\begin{array}{r}3.751 \mathrm{E}- \\
02\end{array}$ & $\begin{array}{r}3.751 \mathrm{E}- \\
02\end{array}$ & $\begin{array}{r}7.932 \mathrm{E}- \\
02\end{array}$ & $\begin{array}{r}1.218 \mathrm{E}- \\
01\end{array}$ & $\begin{array}{r}1.393 \mathrm{E}- \\
01\end{array}$ & $\begin{array}{r}1.384 \mathrm{E}- \\
01\end{array}$ & $\begin{array}{r}1.297 \mathrm{E}- \\
01\end{array}$ & $\begin{array}{r}1.188 \mathrm{E}- \\
01\end{array}$ \\
\hline 4 & $\begin{array}{r}1.250 \mathrm{E}- \\
02 \\
\end{array}$ & $\begin{array}{r}1.250 \mathrm{E}- \\
02 \\
\end{array}$ & $\begin{array}{r}4.579 \mathrm{E}- \\
02 \\
\end{array}$ & $\begin{array}{r}9.546 \mathrm{E}- \\
02 \\
\end{array}$ & $\begin{array}{r}1.247 \mathrm{E}- \\
01 \\
\end{array}$ & $\begin{array}{r}1.313 \mathrm{E}- \\
01 \\
\end{array}$ & $\begin{array}{r}1.263 \mathrm{E}- \\
01 \\
\end{array}$ & $\begin{array}{r}1.172 \mathrm{E}- \\
01 \\
\end{array}$ \\
\hline 6 & $\begin{array}{r}2.388 \mathrm{E}- \\
03 \\
\end{array}$ & $\begin{array}{r}2.388 \mathrm{E}- \\
03 \\
\end{array}$ & $\begin{array}{r}2.048 \mathrm{E}- \\
02 \\
\end{array}$ & $\begin{array}{r}6.773 \mathrm{E}- \\
02 \\
\end{array}$ & $\begin{array}{r}1.073 \mathrm{E}- \\
01 \\
\end{array}$ & $\begin{array}{r}1.224 \mathrm{E}- \\
01 \\
\end{array}$ & $\begin{array}{r}1.221 \mathrm{E}- \\
01 \\
\end{array}$ & $\begin{array}{r}1.152 \mathrm{E}- \\
01 \\
\end{array}$ \\
\hline 8 & $\begin{array}{r}1.909 \mathrm{E}- \\
04 \\
\end{array}$ & $\begin{array}{r}1.909 \mathrm{E}- \\
04 \\
\end{array}$ & $\begin{array}{r}6.181 \mathrm{E}- \\
03 \\
\end{array}$ & $\begin{array}{r}4.143 \mathrm{E}- \\
02 \\
\end{array}$ & $\begin{array}{r}8.725 \mathrm{E}- \\
02 \\
\end{array}$ & $\begin{array}{r}1.116 \mathrm{E}- \\
01 \\
\end{array}$ & $\begin{array}{r}1.168 \mathrm{E}- \\
01 \\
\end{array}$ & $\begin{array}{r}1.127 \mathrm{E}- \\
01 \\
\end{array}$ \\
\hline 10 & $\begin{array}{r}3.872 \mathrm{E}- \\
06\end{array}$ & $\begin{array}{r}3.872 \mathrm{E}- \\
06\end{array}$ & $\begin{array}{r}1.011 \mathrm{E}- \\
03\end{array}$ & $\begin{array}{r}2.025 \mathrm{E}- \\
02 \\
\end{array}$ & $\begin{array}{r}6.540 \mathrm{E}- \\
02 \\
\end{array}$ & $\begin{array}{r}9.849 \mathrm{E}- \\
02\end{array}$ & $\begin{array}{r}1.102 \mathrm{E}- \\
01\end{array}$ & $\begin{array}{r}1.096 \mathrm{E}- \\
01 \\
\end{array}$ \\
\hline 12 & $\begin{array}{r}9.006 \mathrm{E}- \\
09 \\
\end{array}$ & $\begin{array}{r}9.006 \mathrm{E}- \\
09 \\
\end{array}$ & $\begin{array}{r}6.338 \mathrm{E}- \\
05 \\
\end{array}$ & $\begin{array}{r}7.010 \mathrm{E}- \\
03 \\
\end{array}$ & $\begin{array}{r}4.344 \mathrm{E}- \\
02 \\
\end{array}$ & $\begin{array}{r}8.310 \mathrm{E}- \\
02 \\
\end{array}$ & $\begin{array}{r}1.021 \mathrm{E}- \\
01 \\
\end{array}$ & $\begin{array}{r}1.056 \mathrm{E}- \\
01 \\
\end{array}$ \\
\hline 14 & $\begin{array}{r}6.810 \mathrm{E}- \\
13 \\
\end{array}$ & $\begin{array}{r}6.810 \mathrm{E}- \\
13 \\
\end{array}$ & $\begin{array}{r}8.756 \mathrm{E}- \\
07 \\
\end{array}$ & $\begin{array}{r}1.421 \mathrm{E}- \\
03 \\
\end{array}$ & $\begin{array}{r}2.406 \mathrm{E}- \\
02 \\
\end{array}$ & $\begin{array}{r}6.571 \mathrm{E}- \\
02 \\
\end{array}$ & $\begin{array}{r}9.219 \mathrm{E}- \\
02 \\
\end{array}$ & $\begin{array}{r}1.007 \mathrm{E}- \\
01 \\
\end{array}$ \\
\hline 16 & $\begin{array}{r}0.000 \mathrm{E}+ \\
00 \\
\end{array}$ & $\begin{array}{r}0.000 \mathrm{E}+ \\
00 \\
\end{array}$ & $\begin{array}{r}1.110 \mathrm{E}- \\
09 \\
\end{array}$ & $\begin{array}{r}1.246 \mathrm{E}- \\
04 \\
\end{array}$ & $\begin{array}{r}1.010 \mathrm{E}- \\
02 \\
\end{array}$ & $\begin{array}{r}4.725 \mathrm{E}- \\
02 \\
\end{array}$ & $\begin{array}{r}8.033 \mathrm{E}- \\
02 \\
\end{array}$ & $\begin{array}{r}9.460 \mathrm{E}- \\
02 \\
\end{array}$ \\
\hline 18 & $\begin{array}{r}0.000 \mathrm{E}+ \\
00 \\
\end{array}$ & $\begin{array}{r}0.000 \mathrm{E}+ \\
00 \\
\end{array}$ & $\begin{array}{r}3.209 \mathrm{E}- \\
14 \\
\end{array}$ & $\begin{array}{r}2.925 \mathrm{E}- \\
06 \\
\end{array}$ & $\begin{array}{r}2.763 \mathrm{E}- \\
03 \\
\end{array}$ & $\begin{array}{r}2.949 \mathrm{E}- \\
02 \\
\end{array}$ & $\begin{array}{r}6.654 \mathrm{E}- \\
02 \\
\end{array}$ & $\begin{array}{r}8.708 \mathrm{E}- \\
02 \\
\end{array}$ \\
\hline 20 & $\begin{array}{r}0.000 \mathrm{E}+ \\
00 \\
\end{array}$ & $\begin{array}{r}0.000 \mathrm{E}+ \\
00 \\
\end{array}$ & $\begin{array}{r}0.000 \mathrm{E}+ \\
00 \\
\end{array}$ & $\begin{array}{r}8.573 \mathrm{E}- \\
09 \\
\end{array}$ & $\begin{array}{r}3.876 \mathrm{E}- \\
04 \\
\end{array}$ & $\begin{array}{r}1.486 \mathrm{E}- \\
02 \\
\end{array}$ & $\begin{array}{r}5.121 \mathrm{E}- \\
02 \\
\end{array}$ & $\begin{array}{r}7.794 \mathrm{E}- \\
02 \\
\end{array}$ \\
\hline 22 & $\begin{array}{r}0.000 \mathrm{E}+ \\
00 \\
\end{array}$ & $\begin{array}{r}0.000 \mathrm{E}+ \\
00 \\
\end{array}$ & $\begin{array}{r}0.000 \mathrm{E}+ \\
00 \\
\end{array}$ & $\begin{array}{r}9.346 \mathrm{E}- \\
13 \\
\end{array}$ & $\begin{array}{r}1.907 \mathrm{E}- \\
05 \\
\end{array}$ & $\begin{array}{r}5.394 \mathrm{E}- \\
03 \\
\end{array}$ & $\begin{array}{r}3.538 \mathrm{E}- \\
02 \\
\end{array}$ & $\begin{array}{r}6.708 \mathrm{E}- \\
02 \\
\end{array}$ \\
\hline 24 & $\begin{array}{r}0.000 \mathrm{E}+ \\
00 \\
\end{array}$ & $\begin{array}{r}0.000 \mathrm{E}+ \\
00 \\
\end{array}$ & $\begin{array}{r}0.000 \mathrm{E}+ \\
00 \\
\end{array}$ & $\begin{array}{r}0.000 \mathrm{E}+0 \\
0\end{array}$ & $\begin{array}{r}1.799 \mathrm{E}- \\
07 \\
\end{array}$ & $\begin{array}{r}1.177 \mathrm{E}- \\
03\end{array}$ & $\begin{array}{r}2.080 \mathrm{E}- \\
02 \\
\end{array}$ & $\begin{array}{r}5.457 \mathrm{E}- \\
02\end{array}$ \\
\hline
\end{tabular}


British Journal of Computer, Networking and Information Technology

ISSN: 2689-5315

Volume 4, Issue 1, 2021 (pp. 13-20)

www.abjournals.org

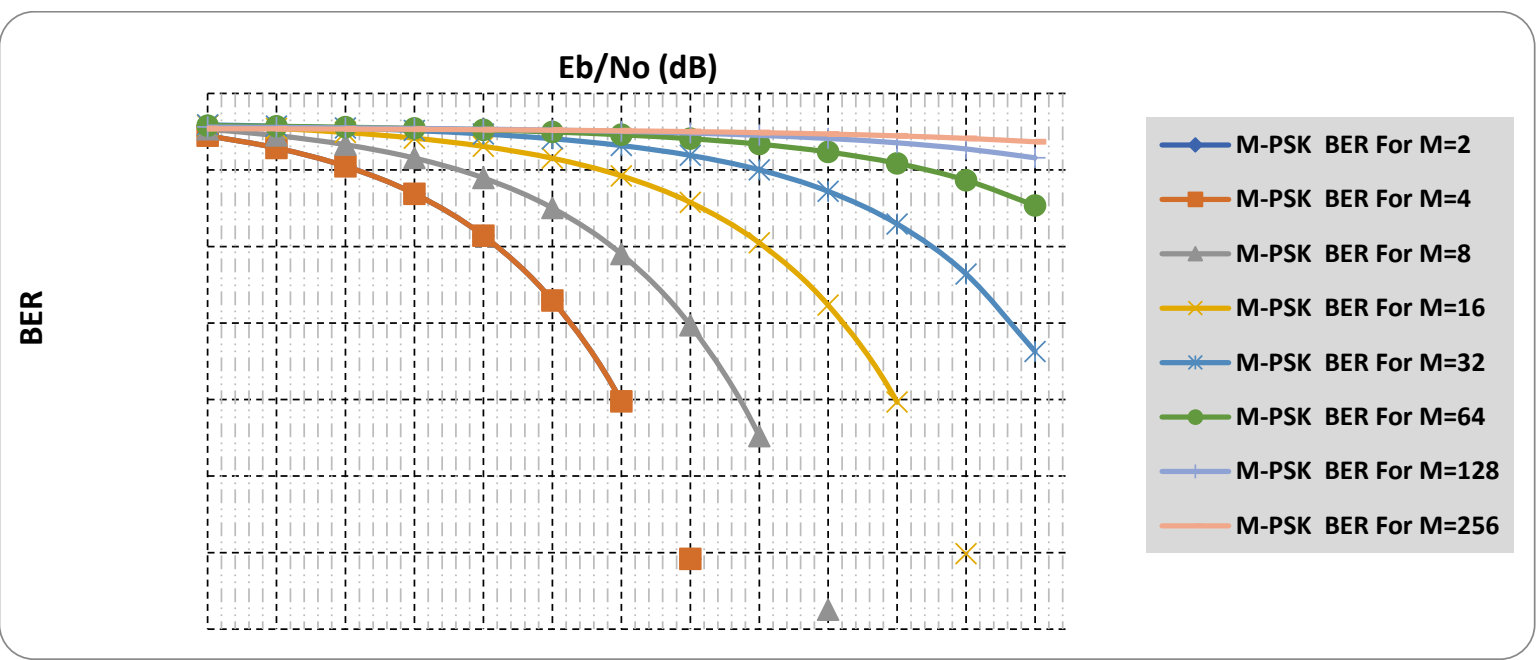

Figure 1 The MPSK modulation BER versus Eb/No (dB)

Table 3 BER Versus $M$ for selected values of $\mathrm{Eb} / \mathrm{No}$

\begin{tabular}{|c|c|c|c|}
\hline $\begin{array}{l}\text { Modulation Order, } \\
\mathrm{M}\end{array}$ & $\begin{array}{l}\text { BER for Eb/No }=4 \\
\mathrm{~dB}\end{array}$ & $\begin{array}{l}\text { BER for Eb/No }=8 \\
\mathrm{~dB}\end{array}$ & $\begin{array}{l}\text { BER for Eb/No }=12 \\
\mathrm{~dB}\end{array}$ \\
\hline $\mathbf{2}$ & $\mathbf{1 . 2 5 0 1 E - 0 2}$ & $\mathbf{1 . 9 0 9 1 E - 0 4}$ & $\mathbf{9 . 0 0 6 0 E - 0 9}$ \\
\hline 4 & $1.2501 \mathrm{E}-02$ & $1.9091 \mathrm{E}-04$ & $9.0060 \mathrm{E}-09$ \\
\hline 8 & $4.5791 \mathrm{E}-02$ & $6.1811 \mathrm{E}-03$ & $6.3379 \mathrm{E}-05$ \\
\hline 16 & $9.5456 \mathrm{E}-02$ & $4.1432 \mathrm{E}-02$ & $7.0096 \mathrm{E}-03$ \\
\hline 32 & $1.2465 \mathrm{E}-01$ & $8.7246 \mathrm{E}-02$ & $4.3443 \mathrm{E}-02$ \\
\hline 64 & $1.3127 \mathrm{E}-01$ & $1.1157 \mathrm{E}-01$ & $8.3101 \mathrm{E}-02$ \\
\hline 128 & $1.2633 \mathrm{E}-01$ & $1.1680 \mathrm{E}-01$ & $1.0210 \mathrm{E}-01$ \\
\hline 256 & $1.1725 \mathrm{E}-01$ & $1.1273 \mathrm{E}-01$ & $1.0563 \mathrm{E}-01$ \\
\hline
\end{tabular}

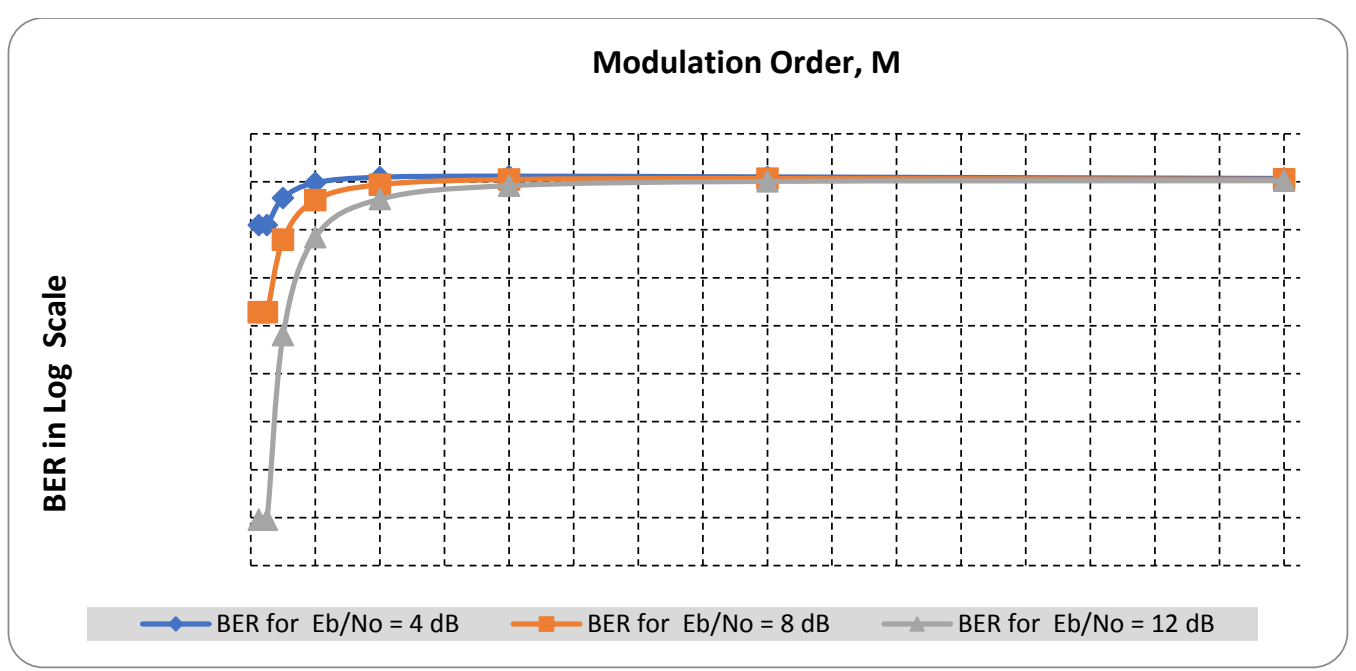

Figure 2 BER Versus $M$ for selected values of $\mathrm{Eb} / \mathrm{No}$ 


\section{CONCLUSION}

A generalized formula for the computation of the MPSK modulation Probability of Bit Error (BER) is presented along with BER expressed in terms of Q function, error function (erf) and complementary error function (erfc). Also, the formula for the symbol error rate of the MPSK modulation scheme is presented. A simulation result of the BER for various modulation order and energy per bit to noise power density ratio $(\mathrm{Eb} / \mathrm{No})$ is presented. The results showed general relationships that exist among modulation order, BER and Eb/No for the MPSK modulation scheme.

\section{REFERENCES}

1. Channi, H. K. (2016). A Comparative Study of Various Digital Modulation Techniques. International Journal in IT and Engineering, 4(03).

2. Faruque, S. (2017). Phase Shift Keying (PSK). In Radio Frequency Modulation Made Easy (pp. 69-83). Springer, Cham.

3. Wang, X. F., AlVARINO, A. R., Gaal, P., Chen, W., Xu, H., Fakoorian, S. A. A., ... \& Wang, R. (2018). U.S. Patent No. 9,948,486. Washington, DC: U.S. Patent and Trademark Office.

4. Radjapov, D. S. (2019). ANALYSIS CREATING AND MODULATION PROCESSES OF BPSK SIGNALS IN MATLAB SOFTWARE. Modern Science, (7-2), 264-268.

5. Chirag, B., Lohith, A., \& Prashantha, H. S. (2017, April). Comparative performance analysis of various digital modulation schemes in AWGN channel. In 2017 Innovations in Power and Advanced Computing Technologies (i-PACT) (pp. 1-5). IEEE.

6. Taqi, A. K. (2016). Implementation of Digital Modulation Technique and Calculate the Bit Error Rate Performance using Matlab.

7. Al Safi, A., \& Bazuin, B. (2016, October). FPGA based implementation of BPSK and QPSK modulators using address reverse accumulators. In 2016 IEEE 7th Annual Ubiquitous Computing, Electronics \& Mobile Communication Conference (UEMCON) (pp. 1-6). IEEE.

8. Elashkar, N. E., Aboudina, M., Fahmy, H. A., Ibrahim, G. H., \& Khalil, A. H. (2016). Memristor based BPSK and QPSK demodulators with nonlinear dopant drift model. Microelectronics Journal, 56, 17-24.

9. Anastasov, J. A., Marjanović, Z. M., Milić, D. N., \& Djordjevic, G. T. (2018). Average BER and noisy reference loss of partially coherent PSK demodulation over shadowed multipath fading channel. IEEE Transactions on Vehicular Technology, 67(8), 78317835.

10. Mahender, K., Anil Kumar, T., \& Ramesh, K. S. (2017). SER and BER performance analysis of digital modulation schemes over multipath fading channels. Journal of Advanced Research in Dynamical and Control Systems, 9(2), 287-291.

11. Liu, X., Wang, Y., Zhou, F., \& Hu, R. Q. (2018, October). BER analysis for NOMAenabled visible light communication systems with M-PSK. In 2018 10th International Conference on Wireless Communications and Signal Processing (WCSP) (pp. 1-7). IEEE.

12. Alodeh, M., Chatzinotas, S., \& Ottersten, B. (2017). Symbol-level multiuser MISO precoding for multi-level adaptive modulation. IEEE Transactions on Wireless Communications, 16(8), 5511-5524. 
13. Wang, H., Pan, L., Lu, F., Murugan, G. S., Sun, Y., \& Ji, Y. (2018). All-optical multilevel phase quantization based on phase-sensitive amplification with low-order harmonics. Journal of Lightwave Technology, 36(24), 5833-5840.

14. Bogris, A., \& Pikasis, E. (2016). Enhanced multi-level phase quantizer for the optical processing of M-PSK signals. Journal of Lightwave Technology, 34(10), 2571-2577.

15. Xing, Z., Wang, H., \& Ji, Y. (2019). QPSK signal regeneration based on vector phase sensitive amplification with low pump powers. IEEE Access, 7, 63936-63943.

16. Faruque, S. (2017). Radio frequency modulation made easy. Springer International Publishing.

17. Mahfuz-Ur-Rahman, A. M., Islam, M. M., \& Islam, M. R. (2016, December). Performance analysis of modulation techniques in multilevel inverters for direct grid connected photovoltaic arrays. In 2016 9th International Conference on Electrical and Computer Engineering (ICECE) (pp. 66-69). IEEE.

18. Biyoghe, J. S. (2017). Design and implementation of a high data rate QPSK demodulator for nanosatellites (Doctoral dissertation, Cape Peninsula University of Technology).

19. Omijeh, B. O., \& Emenike, J. U. Computer-Based Analysis of The Performance of Different Modulation Techniques in Wcdmasystem With different Channels.

20. Haboobi, H., \& Kadhum, M. R. (2019). Impact study and evaluation of higher modulation schemes on physical layer of upcoming wireless mobile networks. Int. J. Adv. Comput. Sci. Appl, 10(5). 